\title{
Bacterial Hydrolysis of Host Glycoproteins - Powerful Protein Modification and Efficient Nutrient Acquisition
}

\author{
Julia Garbe Mattias Collin \\ Department of Clinical Sciences, Division of Infection Medicine, Lund University Biomedical Center B14, \\ Lund, Sweden
}

\section{Key Words}

Glycosylation • Bacteria - Glycan • Hydrolysis •

Endoglycosidase $\cdot$ Exoglycosidase

\begin{abstract}
Glycoproteins are ubiquitous in nature and fundamental to most biological processes, including the human immune system. The glycoprotein carbohydrate moieties, or glycans, are very diverse in their structure and composition, and have major effects on the chemical, physical and biological properties of these glycoproteins. The hydrolysis of glycoprotein glycans by bacterial glycosidases can have dramatic effects on glycoprotein function and, thereby, be beneficial for the bacteria in different ways. This review gives an introduction to the expanding field of extracellular glycosidases from bacterial pathogens with activity on host glycoproteins, describes some known and proposed consequences for the host and the bacteria and discusses some evolutionary and regulatory aspects of bacterial glycosidases.
\end{abstract}

Copyright $\odot 2012$ S. Karger AG, Basel

\section{Introduction}

Covalently attached carbohydrate chains, called glycans, are abundant on proteins within most eukaryotic organisms. Glycans are classified primarily due to the na-

\section{KARGER}

E-Mail karger@karger.ch www.karger.com/jin
() 2012 S. Karger AG, Basel
$1662-811 X / 12 / 0042-0121 \$ 38.00 / 0 \quad$ Karger
This is an Open Access article licensed under the terms of

This is an Open Access article licensed under the terms of
the Creative Commons Attribution- NonCommercial-NoDerivs 3.0 License (www.karger.com/OA-license), applicable to the online version of the article only. Distribution for non-commercial purposes only. ture of their linkage. $\mathrm{N}$-linked glycans begin with an $\mathrm{N}$ acetylglucosamine residue that is attached to an asparagine residue by the recognition of a glycosylation sequence (Asn-X-Ser/Thr), whereas $O$-linked glycans start with an $\mathrm{N}$-acetylgalactosamine that is attached to a serine or a threonine $[1,2]$. The structure of these oligosaccharides is very diverse and plays an important role in the biological function of glycoproteins [2,3]. Indeed, appropriate glycosylation is crucial for cellular functions, hence alterations in glycosylation are associated with certain medical disorders such as autoimmunity and cancer [4, 5]. N-linked glycans have a major role in protein folding, since the glycans are involved in intermolecular proteinglycan interactions and interaction with lectin-type chaperones. But the glycans are also important for correct protein sorting and transport and are therefore crucial for the glycoprotein function [6, 7]. Another essential function of $N$-linked glycans is to promote protein-protein interactions mediating biological responses, e.g. the binding of the immunoglobulin Fc fragment to the Fc receptor $[8,9]$. Interestingly, $N$-linked glycans share a common core structure $\left(\mathrm{Man}_{3} \mathrm{GlcNAc}_{2}\right)$, because they are derived from a block of 14 sugars that is added to the newly synthesized protein in the endoplasmic reticulum. The glycan is then further processed in the endoplasmic reticulum and Golgi complex, which results in the diverse structure of $N$-linked glycans $[3,6]$. In contrast to the $N$ linked glycans, O-linked glycans do not have typical

Dr. Julia Garbe

Department of Clinical Sciences, Division of Infection Medicine

Lund University Biomedical Center B14

SE-221 84 Lund (Sweden)

Tel. +46222 9845, E-Mail julia.garbe@ med.lu.se 
common structural elements except for the GalNAc that is attached to the hydroxyl group of a serine or a threonine. However, the structural diversity, similar to the $N$ linked glycans, is given by the attachment of terminal sugars that are typically sialic acid, fucose, galactose, $\mathrm{N}$ acetylglucosamine or $\mathrm{N}$-acetylgalactosamine. $\mathrm{O}$-linked glycans can be further divided into 5 groups (core 1-5) based on the sugars directly attached to the first GalNAc [3]. O-linked glycans are not as well studied, but it is known that they have important functions in providing charge, water-binding properties as well as stabilizing the glycoprotein [3]. As glycans are very abundant on eukaryotic cells, it is not surprising that these carbohydrate structures are frequent targets for the binding of pathogens and toxins [10]. For instance, glycans play a major role in the adhesion of Helicobacter pylori to gastric epithelial cells [11] and for the binding of influenza A hemagglutinin to sialic acid-containing glycans [12]

Many microbial proteins target components of the immune system and could thereby contribute to immune evasion and successful colonization and/or invasion of the host. For instance, secretion of proteases that degrade proteins of the innate and acquired immune system, such as immunoglobulins or complement factors, is an effective way to interfere with the immune response and is conducted by many bacterial pathogens such as Streptococcus pyogenes, Enterococcus faecalis, Staphylococcus aureus and Pseudomonas aeruginosa [13]. Such immunomodulation can also result from glycan hydrolysis, as almost all proteins from the innate and adaptive immune system are glycosylated and these glycans play important roles in stability and in the immune response itself [14]. In addition, glycans are an important source of nutrients during growth in vivo. Many commensals, especially from the gastrointestinal tract, express glycosidases that metabolize glycans from host glycoproteins, such as mucins $[15,16]$. Nutrient acquisition via deglycosylation of glycoproteins also seems to play an important role in the survival and persistence of pathogens colonizing different niches of the human body $[17,18]$.

Glycoside hydrolases (glycosidases), which have the capability of hydrolyzing sugar residues from glycoproteins, are widespread among many organisms including bacteria and are classified by amino acid sequence similarities [19]. A very good overview of the 125 known glycoside hydrolase families is presented by the online web service CAZY (http://www.cazy.org/Glycoside-Hydrolases.html) initiated by Bernard Henrissat and Gideon Davies [20]. Bacterial glycosidases can be divided into exo- or endoglycosidases, depending on if they hydrolyze sugar residues from the nonreducing end (exoglycosidases) of the sugar chain or if they hydrolyze the sugar chain at defined sites within the glycan (endoglycosidases) (fig. 1, table 1). For more information about the biochemistry of bacterial glycosidases, please read Yamamoto et al. [21].

This short review describes bacterial glycosidases from pathogens that have activity on the glycans of host glycoproteins, mainly from the immune system. Effects such as immunomodulation, nutrient acquisition and improvement of adherence on host cells due to glycosidase activity are described. Finally, we discuss some evolutionary and regulatory aspects of the described glycosidases. As the focus of this review is glycosyl hydrolases, the glycosyl transferases (including sialyltransferases) are not discussed. We are aware that some of the described glycosidases have been shown to have more functions with consequences for the host and the pathogens. We cannot include all relevant information in this short review, but we hope to inspire the readership to further reading and experimentation within this evolving field.

\section{Immunomodulation by Streptococcus pyogenes}

S. pyogenes (group A Streptococcus) is a Gram-positive bacterium that causes uncomplicated infections such as pharyngitis, scarlet fever and impetigo, but can also cause very severe conditions like necrotizing fasciitis and sepsis [22]. S. pyogenes secretes one of the best described bacterial enzymes with glycoside hydrolase activity specific for human IgG, the secreted endo- $\beta$ - $N$-acetylglucosaminidase EndoS [23] (table 1, fig. 1). EndoS contains a family 18 glycoside hydrolase domain and has a size of $108 \mathrm{kDa}$. It selectively cleaves the $\beta 1-4$ linkage between the two $\mathrm{N}$-acetylglucosamines leaving one $\mathrm{N}$-acetylglucosamine on the protein backbone of the $\gamma$-chain of human IgG [23]. Interestingly, EndoS requires the native fold of IgG (suggesting important protein-protein interactions) for glycan-hydrolyzing activity in contrast to the related endoglycosidases EndoF $_{1-3}$ (Elizabethkingia meningoseptica, see below) that require or are enhanced in their activity by denaturation of the glycoprotein $[24,25]$.

IgG and the other immunoglobulins (IgA, IgM, IgD and $\operatorname{IgE}$ ) are the major soluble products of the adaptive immune system and provide the host with long-term immunity against pathogens [9]. IgG is the most abundant immunoglobulin and it contains one conserved $\mathrm{N}$-glycosylation site (Asn-297). The glycan of IgG is crucial for the stability and conformation of the Fc portion. Deglycosyl- 
Fig. 1. Schematic representation of glycan hydrolysis of $\mathrm{N}$-linked and core-1 Olinked glycans. Arrows indicate cleavage sites of glycosidases (discussed in this review): with a solid line $=$ endoglycosidases and with a dotted line = exoglycosidases, with activity only on terminal carbohydrates. Enzyme names in bold indicate cleavage sites (shown or proposed in the references). Other indicated cleavage sites are suggested cleavage sites due to similarities with other enzymes. Please note that EngPA and EngEF also have activity on core-3 O-linked glycans and that EngSP activity probably relies on the cleavage of the sialic acid by NanA. Gal = Galactose; GalNAc = N-acetylgalactosamine; GlcNAc $=N$-acetylglucosamine; Man $=$ mannose; Sia = sialic acid. Symbols and coloring adhere to guidelines devised by the Consortium for Functional Glycomics (www.functionalglycomics.org/static/ consortium/Nomenclature.shtml).
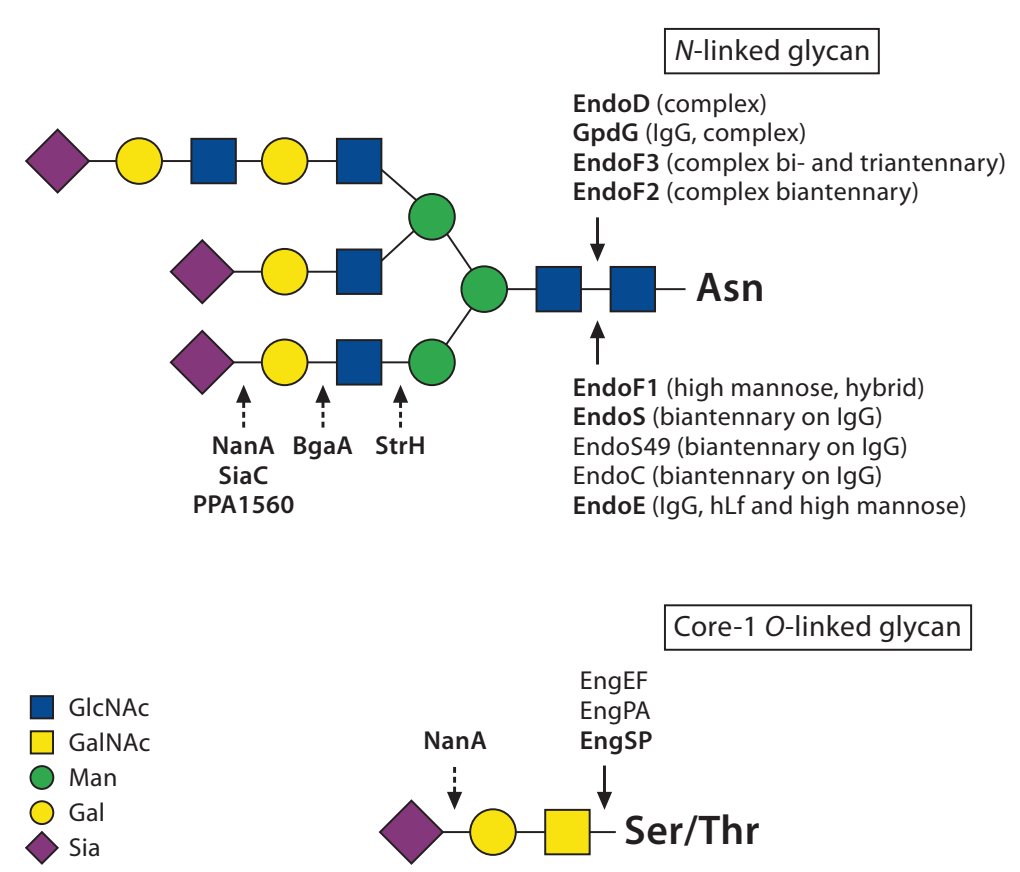

ation of IgG can have consequences such as loss of activation of the complement and loss of binding to Fc receptors $[8,9]$. The activity of EndoS on opsonizing IgG has been shown to lead to a significant reduction in killing of S. pyogenes in blood compared to untreated IgG [26]. The effect of reduced killing of bacteria is due to the decreased ability of IgG to bind to the $\mathrm{Fc}$ receptor of activated monocytes after treatment with EndoS, showing that removal of the glycan from IgG leads to a reduced binding to its receptor [27]. Reduced binding to the receptor leads to a reduced phagocytosis of $S$. pyogenes by monocytes, and therefore to a better survival of the bacteria. Apart from its possible involvement in immune evasion during bacterial colonization/infection, EndoS, due to its specificity for IgG, has successfully been developed as an experimental treatment for antibody-mediated autoimmune diseases, discussed extensively elsewhere [28].

Two other enzymes with family 18 glycoside hydrolase domains and similarities to EndoS were found in streptococci that do not encode the $n d o S$ gene. $\mathrm{EndoS}_{2}$ was discovered in a few $\mathrm{M}$ serotypes of $S$. pyogenes and shares $37.7 \%$ identity on the protein level to EndoS [unpubl. results], whereas the secreted protein EndoC from human and animal isolates of group C streptococci is $67 \%$ identical to EndoS [29]. Both enzymes are able to cleave the $N$ linked glycan of human IgG [unpubl. results]. Based on the similarities to EndoS, EndoS 2 and EndoC most likely cleave the polysaccharide moieties on IgG in a similar way and thereby interfere with IgG-dependent immune responses. The occurrence of EndoS-like enzymes in streptococci that do not express EndoS suggests an important function of these enzymes in streptococci. However, it was recently concluded that EndoS is not a major virulence factor of a highly virulent strain of the M1 serotype in a systemic mouse model [30]. Nevertheless, EndoS hydrolysis of IgG glycans might still be an important mechanism to evade opsonophagocytosis in the human host or to gain nutrients during colonization and localized infections, but this still has to be investigated.

\section{Sequential Deglycosylation of Glycoproteins by Streptococcus pneumoniae}

S. pneumoniae is a Gram-positive bacterium that frequently colonizes the human nasopharynx, a major cause of pneumonia and causes other infections like otitis media, sinusitis and meningitis. S. pneumoniae has evolved complex mechanisms allowing evasion of opsonophagocytic killing in the human host, adherence to host epithelial surfaces and nutrient acquisition by sequential deglycosylation of human glycoproteins. The deglycosylation 
Table 1. Summary of glycosidases and their characteristics described in this review

\begin{tabular}{|c|c|c|c|c|c|c|}
\hline $\begin{array}{l}\text { Glyco- } \\
\text { sidase }\end{array}$ & Organism & $\begin{array}{l}\text { Glycoside } \\
\text { hydrolase family }\end{array}$ & $\begin{array}{l}\text { Endoglycosidase } \\
\text { or exoglycosidase } \\
\text { activity }\end{array}$ & Specificity & $\begin{array}{l}\text { Known glycoprotein } \\
\text { substrate(s) }\end{array}$ & Reference \\
\hline Endos & S. pyogenes & GH 18 & endo & biantennary $N$-linked glycan on IgG & $\operatorname{IgG}$ & {$[23]$} \\
\hline EndoS49 & S. pyogenes & GH 18 & endo & biantennary $N$-linked glycan on $\operatorname{IgG}$ & $\operatorname{IgG}$ & unpublished \\
\hline EndoC & $\begin{array}{l}\text { group C } \\
\text { streptococci }\end{array}$ & GH 18 & endo & biantennary $N$-linked glycan on IgG & $\operatorname{IgG}$ & unpublished \\
\hline NanA & S. pneumoniae & GH 33 & exo & $\begin{array}{l}\text { sialic acid } \alpha 2-3 \text { or } \alpha 2-6 \text { linked to } \\
\text { galactose }\end{array}$ & $\begin{array}{l}\text { IgA1, hLF, hSC, } \\
\alpha 1 \text {-acid glycoprotein }\end{array}$ & {$[17,33,36]$} \\
\hline BgaA & S. pneumoniae & GH 2 & exo & $\begin{array}{l}\text { galactose } \beta 1-4 \text { linked to } \\
N \text {-acetylglucosamine }\end{array}$ & $\begin{array}{l}\text { IgA1, hLF, hSC, } \\
\alpha 1 \text {-acid glycoprotein }\end{array}$ & {$[17,34,36]$} \\
\hline StrH & S. pneumoniae & GH 20 & exo & $\begin{array}{l}N \text {-acetylglucosamine } \beta 1 \text { linked to } \\
\text { mannose }\end{array}$ & $\begin{array}{l}\text { IgA1, hLF, hSC, } \\
\alpha 1 \text {-acid glycoprotein }\end{array}$ & {$[17,35,36]$} \\
\hline EndoD & S. pneumoniae & GH 85 & endo & Complex $N$-linked glycans & TF, fetuin, IgG & {$[40,41]$} \\
\hline EngSP & S. pneumoniae & GH 101 & endo & core-1 $O$-linked glycans & fetuin & {$[44,45]$} \\
\hline EndoE & E. faecalis & $\begin{array}{l}\text { GH } 18 \text { ( } \alpha \text {-domain), } \\
\text { GH } 20 \text { ( } \beta \text {-domain })\end{array}$ & endo & $\begin{array}{l}\text { biantennary and high mannose } N \text {-linked } \\
\text { glycan }(\alpha \text {-domain), biantennary } N \text {-linked } \\
\text { glycan on IgG }(\beta \text {-domain) }\end{array}$ & RNaseB, hLF, IgG & $\begin{array}{l}{[53]} \\
\text { unpublished }\end{array}$ \\
\hline EngEF & E. faecalis & GH 101 & endo & core- 1 and core- $3 O$-linked glycans & unknown & {$[44]$} \\
\hline PPA1560 & P. acnes & GH 33 & exo & unknown & unknown & - \\
\hline EngPA & P. acnes & GH 101 & endo & core- 1 and core- 3 O-linked glycans & unknown & {$[44]$} \\
\hline EndoF $_{1}$ & E. meningoseptica ${ }^{\mathrm{b}}$ & GH 18 & endo & $\begin{array}{l}\text { high mannose, hybrid type } N \text {-linked } \\
\text { glycans }\end{array}$ & $\begin{array}{l}\text { several denatured } \\
\text { glycoproteins }\end{array}$ & {$[58]$} \\
\hline EndoF $_{2}$ & E. meningoseptica ${ }^{\mathrm{b}}$ & GH 18 & endo & complex biantennary $N$-linked glycans & $\begin{array}{l}\text { several denatured } \\
\text { glycoproteins }\end{array}$ & {$[71]$} \\
\hline EndoF $_{3}$ & E. meningoseptica ${ }^{\mathrm{b}}$ & GH 18 & endo & $\begin{array}{l}\text { complex bi- and triantennary } N \text {-linked } \\
\text { glycans }\end{array}$ & $\begin{array}{l}\text { several denatured } \\
\text { glycoproteins }\end{array}$ & {$[71]$} \\
\hline $\mathrm{SiaC}$ & C. canimorsus & GH 33 & exo & unknown & IgG, fetuin & [49] \\
\hline GpdG & C. canimorsus & GH 18 & endo & complex $N$-linked glycans & IgG, fetuin & [49] \\
\hline $\mathrm{NanH}$ & V. cholerae & GH 33 & exo & sialic acid from higher-order gangliosides & $\mathrm{GM}_{1}$ ganglioside & {$[65]$} \\
\hline
\end{tabular}

${ }^{a}$ According to the classification of CAZY (http://www.cazy.org/Glycoside-Hydrolases.html). ${ }^{\mathrm{b}}$ Formerly known as Flavobacterium meningosepticum. $\mathrm{TF}=$ Transferrin, $\mathrm{hSC}=$ human secretory component, $\mathrm{hLF}=$ human lactoferrin.

is accomplished by exo- and endoglycosidases specific to $\mathrm{N}$ - and $\mathrm{O}$-linked glycans. Many glycosidases from $S$. pneumoniae have been identified and many of these have been studied in detail. Since we cannot include all known pneumococcal enzymes and their functions, we refer to an excellent review published recently by S.J. King [31].

The neuraminidase A ( Nan A) is a well-characterized, surface-associated virulence factor of $S$. pneumoniae that catalyzes the release of sialic acid that is $\alpha 2-3$ - or $\alpha 2-6$ linked to galactose [32, 33]. BgaA (247 kDa, GH2), on the other hand, has $\beta$-galactosidase activity and is responsi- ble for the removal of terminally linked galactose that is $\beta 1-4$-linked to $\mathrm{N}$-acetylglucosamine [34]. StrH is a 144$\mathrm{kDa} \beta-N$-acetylglucosaminidase with a family 20 glycoside hydrolase domain that removes terminally linked $N$ acetylglucosamines from human glycoproteins which are $\beta 1$-linked to mannose [35] (fig. 1, table 1). The combined activity of these LPXTG cell wall-anchored exoglycosidases enables $S$. pneumoniae to sequentially deglycosylate human glycoproteins such as IgA1, human lactoferrin (hLF), human secretory component (hSC) and $\alpha 1$-acid glycoprotein $[17,36]$. S. pneumoniae is also able to utilize 
complex $N$-linked glycans from the human glycoprotein $\alpha 1$-acid glycoprotein as a carbon source in vitro, suggesting that it gains nutrients in vivo by glycoprotein hydrolysis [17]. The $\alpha 1$-acid glycoprotein is an acute-phase protein with highly sialylated complex-type $N$-linked glycans which increase in concentration in response to tissue injury, inflammation and infection [37]. Interestingly, $\alpha 1$-acid glycoprotein also has immunomodulatory functions, such as the inactivation of platelet aggregation that is dependent on the sialic acids of $\alpha 1$-acid glycoprotein [38]. But it is unknown if the deglycosylation of $\alpha 1$-acid glycoprotein diminishes its function. The activity of S. pneumoniae on the host airway defense molecules hLF, hSC and IgA1 has been suggested to contribute to the persistence of $S$. pneumoniae in the nasopharynx [36].

Moreover, the combined activities of the above-mentioned S. pneumoniae exoglycosidases promote resistance to opsonophagocytic killing [39]. The complement component $\mathrm{C} 3$ coats the pneumococcal surface in vivo, which ensures recognition by neutrophils and finally leads to opsonophagocytosis. It has been shown that the activity of NanA, BgaA and StrH on an until-now unspecified human serum glycoconjugate (not $\operatorname{IgG}$ ) leads to a reduction of $\mathrm{C} 3$ deposition on the bacterial surface and therefore to a decreased clearance function of the host immune system via opsonophagocytosis [39]. Evading opsonophagocytosis most likely increases the survival and persistence of the pathogen within the human host.

$S$. pneumoniae also expresses an endo- $\beta-N$-acetylglucosaminidase, named EndoD, with a size of $178 \mathrm{kDa}$ and $32 \%$ sequence identity to the glycosidase EndoA from the soil bacterium Arthrobacter protophormiae. EndoD has an LPXTG motif typical for cell wall-anchored proteins and shows activity on the chitobiose core of asparaginelinked oligosaccharides [40]. This endoglycosidase works synergistically with the three pneumococcal exoglycosidases mentioned above to remove complex type oligosaccharides from transferrin, fetuin and $\operatorname{IgG}[40,41]$. The activity on IgG might lead to a similar effect, as shown for EndoS activity [42]. However, it was recently shown that EndoD contains a family 85 glycoside hydrolase domain instead of the family 18 domain found in EndoS. However, GH85 enzymes have a similar configuration of catalytic amino acids as GH18 enzymes, with an asparagine instead of an aspartate residue, and therefore act on the glycosidic linkage between the two $\mathrm{N}$-acetylglucosamines of $N$-linked glycans [43].

Based on the enzyme EngBF from the nonpathogen Bifidobacterium longum, similar endo- $\alpha-N$-acetylgalactosaminidases with GH101 domains were detected in bacteria like S. pneumoniae (EngSP), E. faecalis (EngEF) and Propionibacterium acnes (EngPA), as well as from other nonpathogenic bacteria with amino acid identities between 28 and 49\% [44]. All these enzymes show activity on core-1 $O$-linked glycans. EngSP is surface-associated and acts together with the above-mentioned neuraminidase NanA to hydrolyze sialylated core-1 O-linked glycans. Moreover, it was suggested that this enzyme is common to all $S$. pneumoniae strains. This $O$-glycosidase has an important function for the pathogenesis of $S$. pneumoniae, as it contributes to the adherence to human airway epithelial cells and therefore to the colonization of the upper respiratory tract [45]. The mechanism is not fully understood yet, but it has been suggested that the activity of the glycosidases exposes receptors of $S$. pneumoniae on the epithelial surface [45]. Interestingly, it was also described for NanA that its activity on sialylated glycoconjugates on epithelial cells has a potential role in exposing a receptor for adherence, since a nan $A$ mutant was described as showing a significantly reduced adherence to human airway epithelial cells, than the wild type [36].

\section{Sialidase Activity in Streptococcus oralis}

S. oralis belongs to the normal flora of the oral cavity in humans, but is also associated with systemic diseases such as endocarditis and septicemia. This Gram-positive bacterium is able to utilize the complex-type glycans of human $\alpha 1$-acid glycoprotein as well as mucin to sustain growth $[46,47]$. Several enzymes with sialidase, $N$-acetylglucosaminidase and $\beta$-galactosidase activity were detected in the culture of $S$. oralis supplemented with $\alpha 1$ acid glycoprotein, suggesting that the glycans provide the bacterium with fermentable carbohydrates essential for growth during infection [46]. However, until now, only one secreted sialidase has been purified from the culture medium. It was characterized on protein level as a 144$\mathrm{kDa}$ protein that releases $\mathrm{N}$-acetylneuraminic acid from different glycoconjugates like the above-mentioned $\alpha 1$ acid glycoprotein and bovine submaxillary mucin [48]. As described above in the chapter describing pneumococcal enzymes, $\alpha 1$-acid glycoprotein has an immunomodulatory function. However, whether or not the described sialidase has any effect on the immunomodulatory function of $\alpha 1$-acid glycoprotein is unknown.

This sialidase might also be in complex with other enzymes that have glycosidic and proteolytic activity as described for C. canimorsus [49] (see below), or it works synergistically with other secreted or surface-associated en- 
zymes as does $S$. pneumoniae $[17,36,39]$. Sialidases are not only involved in nutrient acquisition during growth in vivo, they are also defined as a major virulence factor involved in bacterial invasion of the host. It has been suggested that sialidase activity during an infection with $S$. oralis could, for instance, effect the bacterial binding to epithelial cells for host invasion, decrease the half-life of glycoproteins and increase the formation of immune complexes [50]. There is currently no information concerning the genes encoding the described enzymes or when the proteins are expressed. This would be necessary to understand the functions of glycosidases from S. oralis in more detail.

\section{$\mathrm{N}$ - and $\mathrm{O}$-Glycosidases from Enterococcus faecalis}

E. faecalis is a Gram-positive bacterium and a component of the normal flora in the human gastrointestinal tract. Despite the fact that E. faecalis is a well-known member of the microbial consortium in the human gut, it has become a dreaded multiresistant nosocomial pathogen causing infectious endocarditis, urinary tract infections and sepsis $[51,52]$. E. faecalis secretes an endo- $\beta-N$ acetylglucosaminidase (EndoE), identified due to similarities to EndoS. In contrast to EndoS, EndoE (88 kDa) has a unique feature: it combines two enzymatic domains with different glycoside hydrolase activities. The $\alpha$-domain of EndoE contains a family 18 glycoside hydrolase motif and has activity on the $N$-linked mannose-rich glycans of the high-mannose model glycoprotein RNaseB, cleaving the $\beta 1-4$ linkage between the two $\mathrm{N}$-acetylglucosamines. The $\beta$-domain, on the other hand, contains a family 20 glycoside hydrolase domain and is able to release the glycans from the immunoglobulin IgG in the same manner as EndoS [53] (fig. 1, table 1). This activity of EndoE most likely has the same functional consequences for IgG as described for EndoS hydrolysis of IgG. Interestingly, the GH18 $\alpha$-domain of EndoE shows similarities to EndoS while the $\mathrm{GH} 20 \beta$-domain with activity on IgG instead shows similarities to the $\beta-N$-acetylglucosaminidase StrH from S. pneumoniae [35, 53]. However, recent studies showed that the $\alpha$-domain of EndoE has glycosidase activity on the two biantennary $N$-linked glycans of human lactoferrin [unpubl. data]. Lactoferrin and transferrin have multiple biological functions in blood and mucosal surfaces and are considered as components of the innate immune system with antimicrobial activity [54]. The effect of deglycosylation on the function of lactoferrin is at present unknown.
E. faecalis also expresses the endo- $\alpha-N$-acetylgalactosaminidase EngEF with a GH101 domain. As described for EngSP from S. pneumoniae and EngPA from P. acnes, all enzymes of the Eng-family show activity on core-1 $O$-linked glycans, and it was also shown that EngEF from E. faecalis has activity on core-3 O-linked glycans. There is currently no information known about the biological function of EngEF; however, it cannot be excluded that this enzyme contributes to adherence as shown for EngSP from S. pneumoniae [45].

\section{Sialidase and O-Glycosidase Activity of Propionibacterium acnes}

Sialidase activity of the Gram-positive skin commensal and opportunistic pathogen $P$. acnes was described as possibly being involved in degrading host tissues. Three potential sialidases (PPA1560, PPA684 and PPA685) were identified on the genome level, as well as a sialic acid transporter (PPA686), which indicates that $P$. acnes is able to import sialic acids either to gain nutrients or to evade the immune system $[55,56]$. Recently, one sialidase (PPA1560) was characterized and shown to lead to the release of sialic acids from human sebocytes. Strikingly, the loss of sialic acids from the epithelial surface of the sebocytes due to PPA1560 treatment led to an increased interaction of $P$. acnes with the cells, possibly increasing the adhesion of $P$. acnes and thereby sensitizing these cells to $P$. acnes cytotoxicity [57].

Furthermore, $P$. acnes expresses the endo- $\alpha-N$-acetylgalactosaminidase EngPA with a GH101 family domain. Similar to EngEF from E. faecalis, EngPA has activity on core-1 and core-3 O-linked glycans. This enzyme could be involved in adherence, working together with a sialidase (as shown above for EngSP from S. pneumoniae) that acts synergistically with the sialidase NanA to cleave sialylated core-1 O-linked glycans [45].

\section{The Classic $\mathbf{N}$-Glycosidases from Elizabethkingia meningoseptica}

The Gram-negative bacterium E. meningoseptica (formerly known as Flavobacterium meningosepticum) secretes three of the best-characterized endo- $\beta-N$-acetylglucosaminidases $\left(\right.$ EndoF $\left._{1-3}\right)$ that are widely used for the characterization of glycoproteins with $\mathrm{N}$-linked glycans. These enzymes belong to the same family as EndoS from S. pyogenes and EndoE from E. faecalis, with conserved 
family 18 glycosyl hydrolase motifs. Similar to EndoS and EndoE, EndoF ${ }_{1-3}$ cleaves the $\beta 1-4$ linkage between the two $\mathrm{N}$-acetylglucosamines of $\mathrm{N}$-linked glycans, including glycans from IgG, fetuin and $\alpha 1$-acid glycoprotein [58]. As mentioned above, in contrast to the specificity of for instance EndoS and EndoE to IgG, EndoF glycosidases are not specific to the proteins but rather to the glycan structure itself. The differences between $\mathrm{EndoF}_{1-3}$ are their specificities for the type of glycan structure [59]. EndoF $_{1}$ is closely related to EndoH from Streptomyces plicatus and shows activity only on high-mannose and hybrid oligosaccharides, whereas EndoF ${ }_{2}$ and $\mathrm{EndoF}_{3}$ show activity on complex biantennary as well as bi- and triantennary glycans, respectively [60]. Although not well known as a human pathogen, E. meningoseptica can cause meningitis in newborns as well as other infections like pneumonia and endocarditis in immunocompromised patients [61]. The contribution of the 3 mentioned endoglycosidases in the pathogenicity of this bacterium has not been studied.

\section{Glycoprotein Deglycosylation Complex of Capnocytophaga canimorsus}

Another uncommon zoonotic pathogen is the Gramnegative bacterium $C$. canimorsus, which resides in the oral cavity of cats and dogs. Although infections of humans are rare, this bacterium can cause meningitis and fulminant septicemia with a high mortality rate $[49,62]$. C. canimorsus is able to grow in the presence of host glycoproteins from mammalian cells including phagocytes by a mechanism that is dependent on the activity of the sialidase C (SiaC). SiaC does not supply nutrients, but it removes the terminal sialic acids and makes other carbohydrates accessible that can be metabolized by $C$. canimorsus [63]. A mouse model with a SiaC-deficient mutant strain confirmed that $\mathrm{SiaC}$ is a virulence factor that contributes to the persistence of this pathogen [63]. However, $\mathrm{SiaC}$ works together with four outer-membrane lipoproteins $(\mathrm{GpdD}-\mathrm{G})$ and one porin-like protein $(\mathrm{GpdC})$ that are encoded in a so-called polysaccharide utilization locus (PUL). GpdD, E and F probably represent glycanbinding proteins. gpdG encodes an endo- $\beta-N$-acetylglucosaminidase and has activity on IgG and fetuin. The endoglycosidase GpdG cleaves the glycans between the two $\mathrm{N}$-acetylglucosamines and the sugar moiety is then transported into the cell with the help of GpdC, which is very likely a porin of the outer membrane. SiaC, located in the periplasm of this Gram-negative bacterium, cleaves the sialic acid of the glycan and it is assumed that other intracellular enzymes further process the remaining oligosaccharide [49]. The activity on human IgG, as it was also shown for EndoS from S. pyogenes, might contribute not only to nutrient acquisition but could also serve as an immunosuppressive mechanism. However, the molecular mechanism is not yet understood. This is the first report of a glycoprotein deglycosylation complex and the cooperation between different proteins and enzymes to deglycosylate host glycoproteins, which raises the question of whether or not other glycosidases are also part of complex systems.

\section{NanH from Vibrio cholerae Enhances Cholera Toxin Activity}

Another well-investigated sialidase, $\mathrm{NanH}$, is secreted by the Gram-negative intestinal pathogen V. cholerae, which causes the water-borne disease cholera. NanH has a size of $83 \mathrm{kDa}$ and is encoded on the pathogenic island of toxigenic $V$. cholerae strains [64], acts synergistically with the cholera toxin and enhances the severity of the disease [65]. NanH cleaves the sialic acids of higher-order gangliosides and thereby unmasks the receptor of the cholera toxin, $\mathrm{GM}_{1}$. The binding and internalization of the toxin into the epithelial villus is therefore significantly enhanced by the activity of $\mathrm{NanH}$ [65]. However, the gene $n a n H$ is part of a gene cluster, which contains a series of enzymes that might be involved in the utilization of the sialic acids that could be important as a carbon and nitrogen source for $V$. cholerae during infection [64]. $V$. cholerae also produces an endo- $\beta-N$-acetylhexosaminidase that, in combination with a neuraminidase and a protease, is able to degrade mucin. It is thought that the activities of these enzymes help $V$. cholerae to colonize the intestinal epithelium which is covered with sialomucin [66]. Unfortunately, no other studies have been done on the endo- $\beta-N$-acetylhexosaminidase and it cannot be excluded that the neuraminidase activity discovered in the published study can be attributed to $\mathrm{NanH}$.

\section{Phylogenetic Analysis of Bacterial Glycosidases}

The glycosidases described in this review are very diverse and have different functions. In order to obtain some insights into the evolution of these enzymes, we performed a phylogenetic analysis to elucidate if the en- 


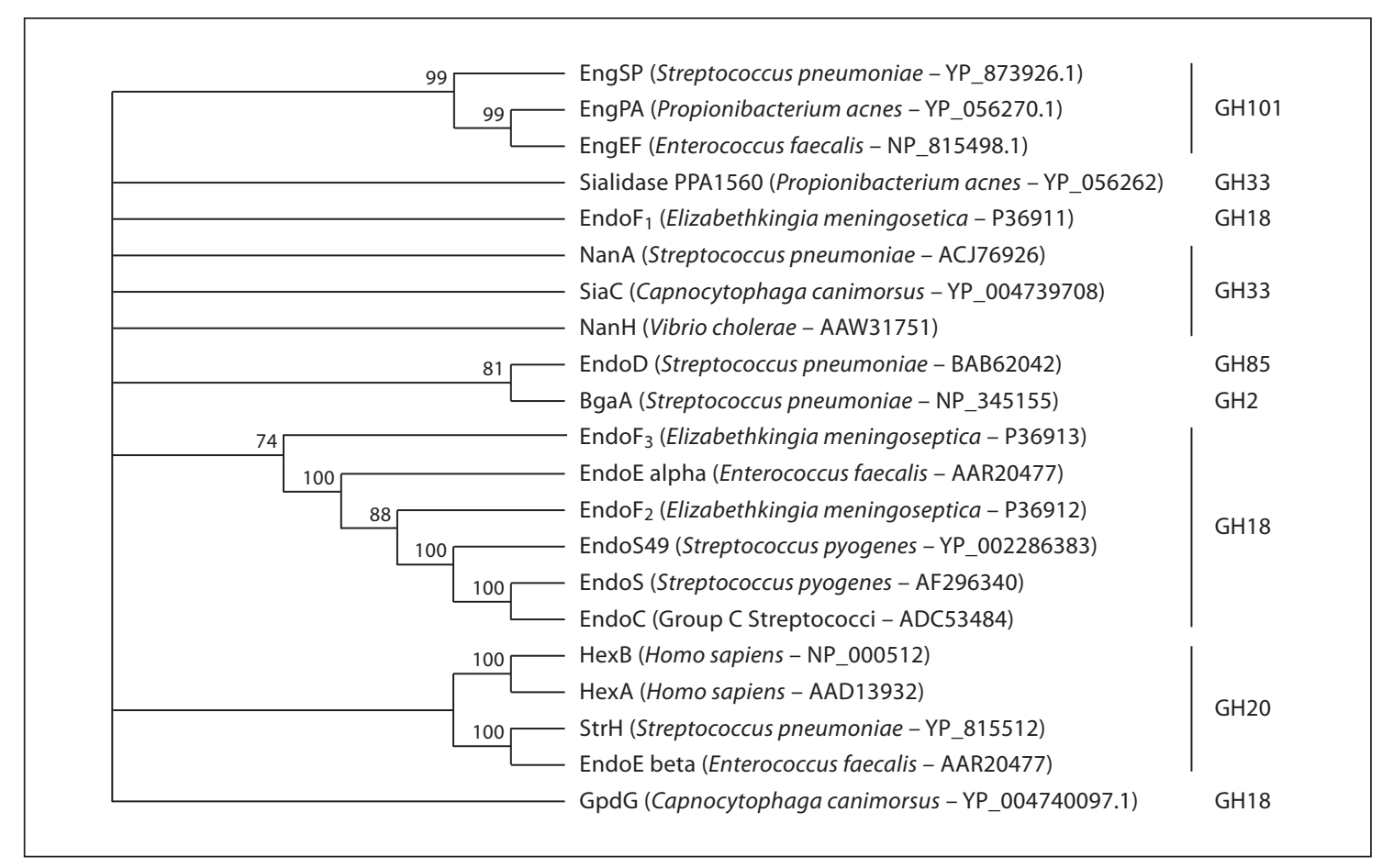

Fig. 2. Phylogenetic analysis of bacterial glycosidases. They were first aligned using the ClustalW algorithm of the MacVector ${ }^{\mathrm{TM}}$ 11.0.2 sequence analysis software suite. Phylogenetic trees were generated using the neighborjoining method, gaps were distributed proportionally with uncorrected $p$ values and were validated by bootstrap analysis (32,000 repetitions). The GenBank accession numbers of the enzymes are indicated in parentheses together with the organism name.

zymes are related and have a common evolutionary background, or if they have evolved independently.

As shown in the phylogenetic analysis in figure 2, several clusters of proteins with similarities can be detected and, not surprisingly, these similarities are mainly based on the glycoside hydrolase domains or functions, respectively. In particular, the enzymes with a GH18 domain like EndoS, EndoC, EndoS $2, \mathrm{EndoF}_{2}$, the $\alpha$-domain of EndoE and $\mathrm{EndoF}_{3}$ cluster together, suggesting that they are closely related. The only two enzymes with a GH18 domain not included in this cluster are EndoF $F_{1}$ and $G p d G$, suggesting that these enzymes have an origin different from the EndoS group. Another enzyme that hydrolyzes the $N$-linked glycans between the two GlcNAc similar to EndoF $_{1}$ and the EndoS group is EndoD, which contains a GH85 domain. As described above, this enzyme has a similar configuration of catalytic amino acids and catalyzes the glycan hydrolysis in a similar way, but probably evolved from a different origin. Another striking feature of all these enzymes is their ability to cleave the $N$-linked glycans of IgG (see also fig. 1, table 1), and it seems very likely that immunomodulation of IgG, the most abundant immunoglobulin, is a very effective way to evade the immune system or even to gain nutrients. However, as the enzymes of different GH families show activity on IgG, it may be, from the evolutionary point of view, an old mechanism of immunomodulation that evolved more than once. The study of the different catalytic mechanisms of these enzymes could help the understanding of their evolutionary background.

The $\beta$-domain of EndoE from E. faecalis and StrH from $S$. pneumoniae share a GH20 domain and cluster together. It has previously been shown that enzymes with a GH20 domain are highly conserved and that they have a common evolutionary origin [67]. We included two human hexosaminidases (HexA and HexB) with GH20 domains to our phylogenetic analysis and we could show that the $\beta$-domain of EndoE and StrH are related to the human enzymes, underscoring their common evolutionary background. Furthermore, this might indicate that host genes could have at some point during coevolution been horizontally transferred to the colonizing bacteria. 
The Eng-family of glycosidases (GH101) that has activity on $O$-linked glycans and which can be found in different pathogenic as well as nonpathogenic bacteria, cluster together because they share a conserved GH101 domain and have similar activities on $O$-linked glycans. Nevertheless, all identified enzymes with a GH101 domain are present only in bacteria, indicating that they might be unique to bacteria and play an important role in the bacterial lifestyle. $O$-glycosidases could be important not only to contribute to the pathogenesis of the bacteria, but also to gain nutrients from glycoproteins such as mucins. Interestingly, the sialidases that share a GH33 domain do not cluster together, suggesting that these enzymes are very diverse and might have a different evolutionary origin. A possible explanation could be that sialic acids are present on the terminal ends of glycans that are very diverse and could therefore have led to the evolutionary diversification of sialidases.

\section{Regulation of Glycosidase Expression}

Very little is known about the regulation of extracellular glycosidases in bacteria. More knowledge could help us to understand the importance of glycosidases for the bacterium during colonization and infection. For instance, it was shown that the genes encoding EndoS and EndoE have so-called catabolite response elements in their promoters, indicating that they are regulated via carbon catabolite repression (CCR) $[68,69]$. CCR is a major regulatory system in bacteria and regulates the expression of genes involved in the use of carbon sources. Recently, it was suggested that there is a close link between basic metabolic processes and the pathogenesis of bacteria because several known virulence factors in bacteria are under the control of the CCR system [69]. Regulation via a major regulatory system like CCR indicates that the enzymes are key molecules in the life of bacteria.

Moreover, there is evidence that the glycan itself can trigger the expression of genes that encode proteins in- volved in glycan metabolism. It has been shown for the human gut bacterium, Bacterioides thetaiotaomicron, that $O$ - and $N$-linked glycans from mucin induce the expression of several PULs [70]. As described for C. canimorsus, PULs encode a complex system of enzymes involved in the deglycosylation of host glycoproteins [49]. Many PULs also contain a sensory regulator, and it is assumed that this regulator can sense glycans and induce the expression of genes involved in the metabolism of glycans [70].

\section{Concluding Remarks}

A large number of known or putative glycosyl hydrolase genes can be found in the genomes of both bacterial pathogens and commensals. The high abundance and, in many cases, high level of conservation indicate that these enzymes are important molecules for colonizing and infecting the human host. The immunomodulation of host proteins by single enzymes or even by protein complexes is widely distributed among bacteria and is very diverse. Enzymes with activity on the glycans of key proteins from the immune system could contribute to the pathogenesis of these bacteria either by evading the host response, or in a more subtle way, by gaining nutrients and/ or supporting the survival in the human body.

The field of glycosidases from pathogens with activity on human glycoproteins is developing but should be expanded in relation to the enzymes from nonpathogenic bacteria colonizing the host. It could be very interesting to investigate if the glycosidases of commensals also have related activities and to elucidate how similar they are to the enzymes from pathogens, structurally and functionally. Such comparisons could potentially reveal how these enzymes evolved with respect to selection pressure originating from the immune system and/or from nutrient limitation. Bacterial enzymes with activity on $O$-linked glycans, represented by the Eng-family of glycosidases, are most likely just the tip of the iceberg with many more to be discovered in both pathogens and commensals.

\section{References}

Bacterial Hydrolysis of Host

Glycoproteins
1 Kobata A: Structures and functions of the sugar chains of glycoproteins. Eur J Biochem 1992;209:483-501.

-2 Dwek RA: Glycobiology: Toward understanding the function of sugars. Chem Rev 1996;96:683-720.

-3 Paulson J: Glycoproteins: what are the sugar chains for? Trends Biochem Sci 1989;272276.
-4 Kim YJ, Varki A: Perspectives on the significance of altered glycosylation of glycoproteins in cancer. Glycoconj J 1997;14:569-576.

-5 Takai T: Roles of Fc receptors in autoimmunity. Nat Rev Immunol 2002;2:580-592.

-6 Helenius A: Intracellular functions of Nlinked glycans. Science 2001;291:2364-2369.

7 Rasmussen J: Effect of glycosylation on protein function. Curr Opin Struct Biol 1992;2: 682-686

Glycoproteins

mmun 2012;4:121-131 
8 Mimura Y, Church S, Ghirlando R, Ashton PR, Dong S, Goodall M, et al: The influence of glycosylation on the thermal stability and effector function expression of human IgG1Fc: properties of a series of truncated glycoforms. Mol Immunol 2000;37:697-706.

$\checkmark 9$ Arnold JN, Wormald MR, Sim RB, Rudd PM, Dwek RA: The impact of glycosylation on the biological function and structure of human immunoglobulins. Annu Rev Immunol 2007;25:21-50.

10 Karlsson KA: Microbial recognition of target-cell glycoconjugates. Curr Opin Struct Biol 1995; 5:622-635.

-11 Magalhaes A, Gomes J, Ismail MN, Haslam SM, Mendes N, Osorio H, et al: Fut2-null mice display an altered glycosylation profile and impaired BabA-mediated Helicobacter pylori adhesion to gastric mucosa. Glycobiology 2009;19:1525-1536.

-12 Weis W, Brown JH, Cusack S, Paulson JC, Skehel JJ, Wiley DC: Structure of the influenza virus haemagglutinin complexed with its receptor, sialic acid. Nature 1988;333: 426-431.

13 Potempa J, Pike RN: Corruption of innate immunity by bacterial proteases. J Innate Immun 2009; 1:70-87.

14 Rudd PM, Elliott T, Cresswell P, Wilson IA, Dwek RA: Glycosylation and the immune system. Science 2001;291:2370-2376.

15 Falk P, Hoskins LC, Larson G: Bacteria of the human intestinal microbiota produce glycosidases specific for lacto-series glycosphingolipids. J Biochem 1990;108:466-474.

$\checkmark 16$ Hooper LV: Commensal host-bacterial relationships in the gut. Science 2001;292:11151118.

17 Burnaugh AM, Frantz LJ, King SJ: Growth of Streptococcus pneumoniae on human glycoconjugates is dependent upon the sequential activity of bacterial exoglycosidases. J Bacteriol 2007;190:221-230.

- 18 Roberts G, Tarelli E, Homer KA, PhilpottHoward J, Beighton D: Production of an endo- $\beta$-N-acetylglucosaminidase activity mediates growth of Enterococcus faecalis on a high-mannose-type glycoprotein. J Bacteriol 2000;182:882-890.

19 Henrissat B: A classification of glycosyl hydrolases based on amino acid sequence similarities. Biochem J 1991;280:309-316.

20 Davies GJ, Gloster TM, Henrissat B: Recent structural insights into the expanding world of carbohydrate-active enzymes. Curr Opin Struct Biol 2005;15:637-645.

21 Yamamoto K, Li S, Li Y: Microbial glycosidases; in Ernst B, Hart G, Sinaÿ P (eds): Carbohydrates in Chemistry and Biology. Weinheim, Wiley-VCH, 2000, pp 497-511.

-22 Cunningham MW: Pathogenesis of group A streptococcal infections. Clin Microbiol Rev 2000;13:470-511.

$\checkmark 23$ Collin M, Olsén A: EndoS, a novel secreted protein from Streptococcus pyogenes with endoglycosidase activity on human IgG. EMBO J 2001;20:3046-3055.
24 Collin M, Olsén A: Effect of SpeB and EndoS from Streptococcus pyogenes on human immunoglobulins. Infect Immun 2001;69: 7187-7189.

25 Tarentino AL, Plummer TH: Enzymatic deglycosylation of asparagine-linked glycans: purification, properties, and specificity of oligosaccharide-cleaving enzymes from Flavobacterium meningosepticum. Meth Enzymol 1994;230:44-57.

-26 Collin M, Svensson MD, Sjöholm AG, Jensenius JC, Sjöbring U, Olsén A: EndoS and SpeB from Streptococcus pyogenes inhibit immunoglobulin-mediated opsonophagocytosis. Infect Immun 2002;70:6646-6651.

27 Allhorn M, Olin AI, Nimmerjahn F, Collin M: Human IgG/Fc gamma R interactions are modulated by streptococcal IgG glycan hydrolysis. PLoS One 2008;3:e1413.

28 Allhorn M, Collin M: Sugar-free antibodies - the bacterial solution to autoimmunity? Ann NY Acad Sci 2009;1173:664-669.

29 Collin M, Olsén A: Extracellular enzymes with immunomodulating activities: variations on a theme in Streptococcus pyogenes. Infect Immun 2003;71:2983-2992.

30 Sjögren J, Okumura CY, Collin M, Nizet V, Hollands A: Study of the IgG endoglycosidase EndoS in group A streptococcal phagocyte resistance and virulence. BMC Microbiol 2011;11:120.

31 King SJ: Pneumococcal modification of host sugars: a major contributor to colonization of the human airway? Mol Oral Microbiol 2010;25:15-24.

32 Scanlon K, Diven W, Glew R: Purification and properties of Streptococcus pneumoniae neuraminidase. Enzyme 1989;41:143-150.

33 Cámara M, Boulnois GJ, Andrew PW, Mitchell TJ: A neuraminidase from Streptococcus pneumoniae has the features of a surface protein. Infect Immun 1994;62:3688-3695.

-34 Zähner D, Hakenbeck R: The Streptococcus pneumoniae $\beta$-galactosidase is a surface protein. J Bacteriol 2000;182:5919-5921.

35 Clarke VA, Platt N, Butters TD: Cloning and expression of the $\beta-\mathrm{N}$-acetylglucosaminidase gene from Streptococcus pneumoniae. J Biol Chem 1995;270:8805-8814.

36 King SJ, Hippe KR, Weiser JN: Deglycosylation of human glycoconjugates by the sequential activities of exoglycosidases expressed by Streptococcus pneumoniae. Mol Microbiol 2006;59:961-974.

>37 Hochepied T, Berger FG, Baumann H, Libert C: $\alpha(1)$-acid glycoprotein: an acute phase protein with inflammatory and immunomodulating properties. Cytokine Growth Factor Rev 2003;14:25-34.

>38 Costello M, Fiedel BA, Gewurz H: Inhibition of platelet aggregation by native and desialised $\alpha-1$ acid glycoprotein. Nature 1979; 281:677-678.
39 Dalia AB, Standish AJ, Weiser JN: Three surface exoglycosidases from Streptococcus pneumoniae, NanA, BgaA, and StrH, promote resistance to opsonophagocytic killing by human neutrophils. Infect Immun 2010; 78:2108-2116.

40 Muramatsu H, Tachikui H, Ushida H, Song X, Qiu Y, Yamamoto S, et al: Molecular cloning and expression of endo- $\beta-\mathrm{N}$-acetylglucosaminidase $\mathrm{D}$, which acts on the core structure of complex type asparagine-linked oligosaccharides. J Biochem 2001;129:923928.

41 Muramatsu T, Koide N, Maeyama K: Further studies on endo- $\beta-\mathrm{N}$-acetylglucosaminidase D1. J Biochem 1978;83:363-370.

42 Koide N, Nose M, Muramatsu T: Recognition of IgG by Fc receptor and complement: effects of glycosidase digestion. Biochem Biophys Res Commun 1977;75:838-844.

43 Abbott DW, Macauley MS, Vocadlo DJ, Boraston AB: Streptococcus pneumoniae endohexosaminidase $\mathrm{D}$, structural and mechanistic insight into substrate-assisted catalysis in family 85 glycoside hydrolases. J Biol Chem 2009;284:11676-11689.

44 Koutsioulis D, Landry D, Guthrie EP: Novel endo- $\alpha-\mathrm{N}$-acetylgalactosaminidases with broader substrate specificity. Glycobiology 2008;18:799-805.

45 Marion C, Limoli DH, Bobulsky GS, Abraham JL, Burnaugh AM, King SJ: Identification of a pneumococcal glycosidase that modifies O-linked glycans. Infect Immun 2009;77:1389-1396.

-46 Byers HL, Tarelli E, Homer KA, Beighton D: Sequential deglycosylation and utilization of the N-linked, complex-type glycans of human alpha1-acid glycoprotein mediates growth of Streptococcus oralis. Glycobiology 1999;9:469-479.

-47 Homer KA, Kelley S, Hawkes J, Beighton D, Grootveld MC: Metabolism of glycoproteinderived sialic acid and $\mathrm{N}$-acetylglucosamine by Streptococcus oralis. Microbiology 1996; 142:1221-1230.

48 Byers HL, Tarelli E, Homer KA, Beighton D: Isolation and characterisation of sialidase from a strain of Streptococcus oralis. J Med Microbiol 2000;49:235-244.

49 Renzi F, Manfredi P, Mally M, Moes S, Jenö P, Cornelis GR: The N-glycan glycoprotein deglycosylation complex (Gpd) from Capnocytophaga canimorsus deglycosylates human IgG. PLoS Pathog 2011;7:e1002118.

50 Corfield T: Bacterial sialidases-roles in pathogenicity and nutrition. Glycobiology 1992;2:509-521.

-51 Fisher K, Phillips C: The ecology, epidemiology and virulence of Enterococcus. Microbi-

ology 2009;155:1749-1757.
52 Sava IG, Heikens E, Huebner J: Pathogenesis and immunity in enterococcal infections. Clin Microbiol Infect 2010;16:533-540. 
53 Collin M, Fischetti VA: A novel secreted endoglycosidase from Enterococcus faecalis with activity on human immunoglobulin $\mathrm{G}$ and ribonuclease B. J Biol Chem 2004;279: 22558-22570.

54 Legrand D, Elass E, Pierce A, Mazurier J: Lactoferrin and host defence: an overview of its immuno-modulating and anti-inflammatory properties. Biometals 2004;17:225229.

55 Brüggemann $\mathrm{H}$ : Insights in the pathogenic potential of Propionibacterium acnes from its complete genome. Semin Cutan Med Surg 2005;24:67-72.

56 Severi E, Hood DW, Thomas GH: Sialic acid utilization by bacterial pathogens. Microbiology 2007;153:2817-2822.

57 Nakatsuji T, Liu Y-T, Huang C-P, Zouboulis CC, Gallo RL, Huang C-M: Vaccination targeting a surface sialidase of $P$. acnes: implication for new treatment of acne vulgaris. PLoS One 2008;3:e1551.

-58 Elder JH, Alexander S: Endo- $\beta$-N-acetylglucosaminidase F: endoglycosidase from Flavobacterium meningosepticum that cleaves both high-mannose and complex glycoproteins. Proc Natl Acad Sci USA 1982;79:45404544.

-59 Waddling CA, Plummer TH, Tarentino AL, Van Roey P: Structural basis for the substrate specificity of endo- $\beta$-N-acetylglucosaminidase F(3). Biochemistry 2000;39:7878-7885.
60 Trimble RB, Tarentino AL: Identification of distinct endoglycosidase (endo) activities in Flavobacterium meningosepticum: endo F1, endo F2, and endo F3. Endo F1 and endo $\mathrm{H}$ hydrolyze only high-mannose and hybrid glycans. J Biol Chem 1991;266:1646-1651.

61 Chiu CH, Waddingdon M, Greenberg D, Schreckenberger PC, Carnahan AM: Atypical Chryseobacterium meningosepticum and meningitis and sepsis in newborns and the immunocompromised, Taiwan. Emerging Infect Dis 2000;6:481-486.

62 Bobo R, Newton E: A previously undescribed gram-negative bacillus causing septicemia and meningitis. Am J Clin Pathol 1976;65: 564-569.

63 Mally M, Shin H, Paroz C, Landmann R, Cornelis GR: Capnocytophaga canimorsus: a human pathogen feeding at the surface of epithelial cells and phagocytes. PLoS Pathog 2008;4:e1000164.

64 Jermyn WS, Boyd EF: Characterization of a novel Vibrio pathogenicity island (VPI-2) encoding neuraminidase (nanH) among toxigenic Vibrio cholerae isolates. Microbiology 2002;148:3681-3693.
65 Galen JE, Ketley JM, Fasano A, Richardson SH, Wasserman SS, Kaper JB: Role of Vibrio cholerae neuraminidase in the function of cholera toxin. Infect Immun 1992;60:406.

66 Stewart-Tull DE, Ollar RA, Scobie TS: Studies on the Vibrio cholerae mucinase complex. I. Enzymic activities associated with the complex. J Med Microbiol 1986;22:325-333.

67 Somerville CC, Colwell RR: Sequence analysis of the $\beta-\mathrm{N}$-acetylhexosaminidase gene of Vibrio vulnificus: evidence for a common evolutionary origin of hexosaminidases. Proc Natl Acad Sci USA 1993;90:6751-6755.

68 Opsata M, Nes IF, Holo H: Class IIa bacteriocin resistance in Enterococcus faecalis V583: the mannose PTS operon mediates global transcriptional responses. BMC Microbiol 2010;10:224.

69 Shelburne SA, Keith D, Horstmann N, Sumby P, Davenport MT, Graviss EA, et al: A direct link between carbohydrate utilization and virulence in the major human pathogen group A Streptococcus. Proc Natl Acad Sci USA 2008;105:1698-1703.

70 Martens E, Chiang HC, Gordon JI: Mucosal glycan foraging enhanced fitness and transmission of a saccharolytic human gut bacterial symbiont. Cell Host Microbe 2008;4: 447-457.

71 Plummer TH Jr, Tarentino AL: Purification of the oligosaccharide-cleaving enzymes of Flavobacterium meningosepticum. Glycobiology 1991;1:257-263. 